Corrigendum

\title{
Corrigendum to "Resveratrol-Enriched Rice Attenuates UVB-ROS-Induced Skin Aging via Downregulation of Inflammatory Cascades"
}

\author{
Lalita Subedi $\mathbb{D}^{1},{ }^{1}$ Taek Hwan Lee, ${ }^{2}$ Hussain Mustatab Wahedi, ${ }^{1,3}$ So-Hyeon Baek, ${ }^{4}$ \\ and Sun Yeou Kim ${ }^{1}{ }^{1}$ \\ ${ }^{1}$ Laboratory of Pharmacognosy, College of Pharmacy and Gachon Institute of Pharmaceutical Sciences, Gachon University, \\ Incheon 21936, Republic of Korea \\ ${ }^{2}$ College of Pharmacy, Yonsei University, No. 162-1, Songdo-dong, Yeonsu-gu, Incheon 406-840, Republic of Korea \\ ${ }^{3}$ Department of Biochemistry, Faculty of Biological Sciences, Quaid-i-Azam University, Islamabad, Pakistan \\ ${ }^{4}$ Department of Well-being Resources, Sunchon National University, Sunchon, Jeonnam 57922, Republic of Korea
}

Correspondence should be addressed to Sun Yeou Kim; sunnykim@gachon.ac.kr

Received 12 December 2017; Accepted 19 December 2017; Published 20 February 2018

Copyright (C) 2018 Lalita Subedi et al. This is an open access article distributed under the Creative Commons Attribution License, which permits unrestricted use, distribution, and reproduction in any medium, provided the original work is properly cited.

In the article titled "Resveratrol-Enriched Rice Attenuates UVB-ROS-Induced Skin Aging via Downregulation of Inflammatory Cascades" [1], the affiliation of the fourth author was incorrect. The corrected authors' list and affiliations are shown above.

\section{References}

[1] L. Subedi, T. H. Lee, H. M. Wahedi, S.-H. Baek, and S. Y. Kim, "Resveratrol-enriched rice attenuates UVB-ROS-induced skin aging via downregulation of inflammatory cascades," Oxidative Medicine and Cellular Longevity, vol. 2017, Article ID 8379539, 15 pages, 2017. 


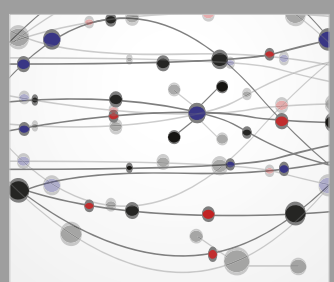

The Scientific World Journal
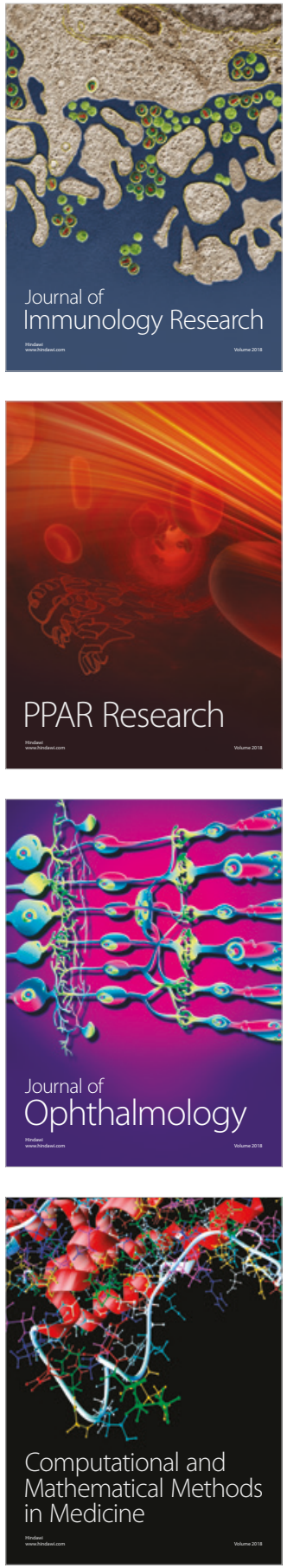

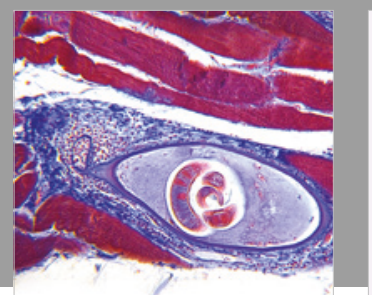

Gastroenterology Research and Practice

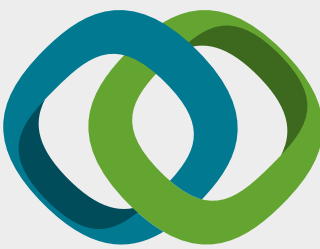

\section{Hindawi}

Submit your manuscripts at

www.hindawi.com
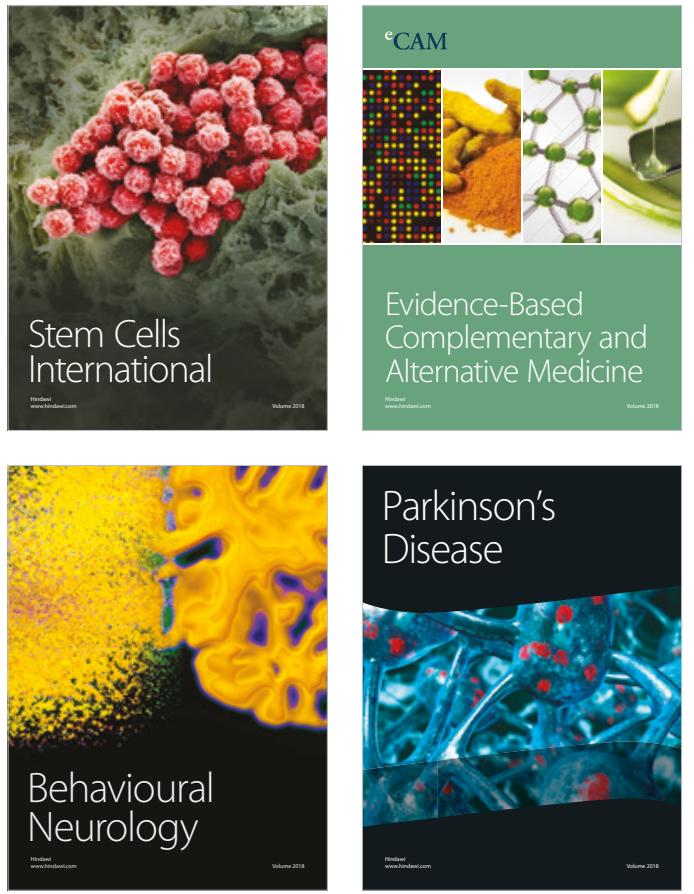

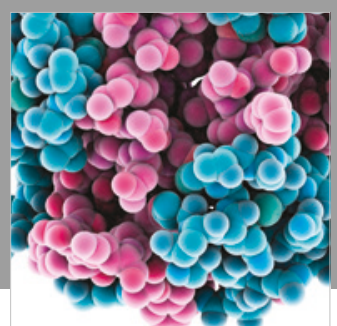

ournal of

Diabetes Research

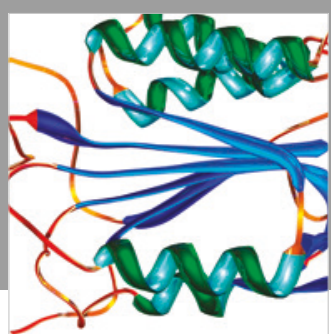

Disease Markers
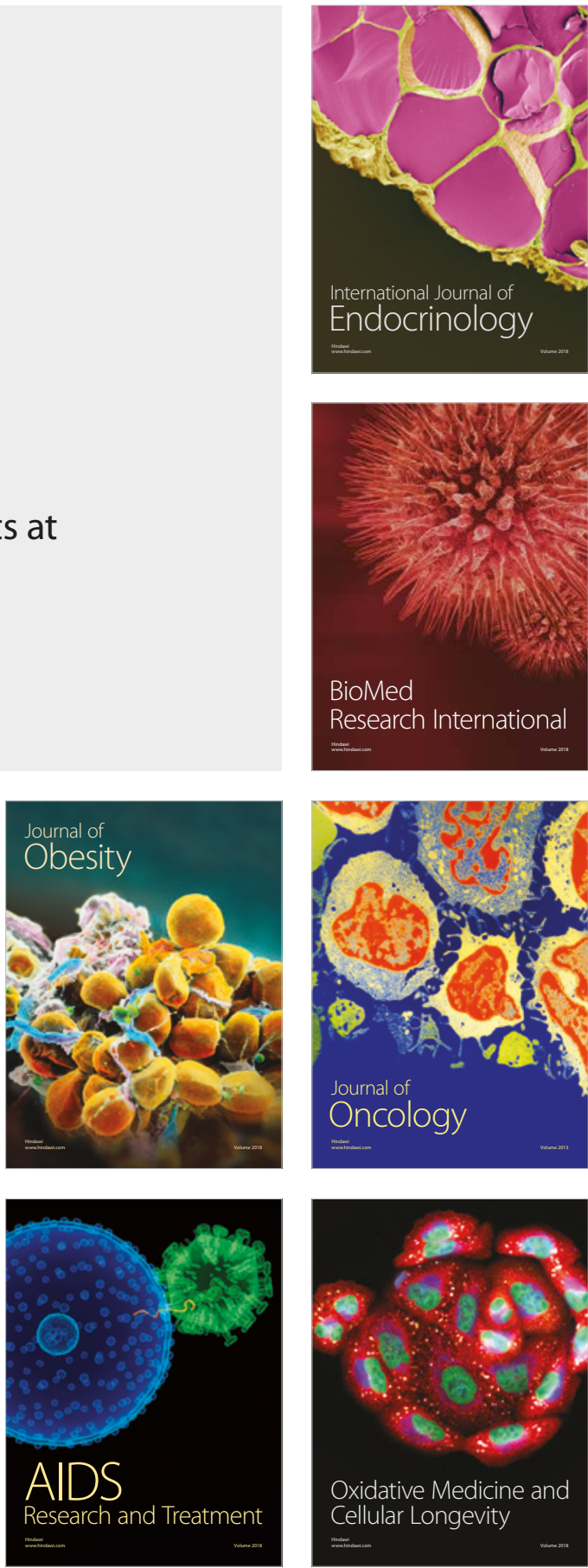